\title{
Honeycomb-serpentine silicon platform for reconfigurable electronics
}

Cite as: Appl. Phys. Lett. 115, 112105 (2019); https://doi.org/10.1063/1.5111018

Submitted: 22 May 2019 . Accepted: 05 August 2019 . Published Online: 10 September 2019

A. N. Damdam, N. Qaisar, and M. M. Hussain

\section{COLLECTIONS}

F This paper was selected as Featured
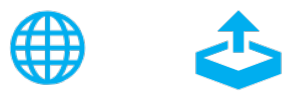

\section{ARTICLES YOU MAY BE INTERESTED IN}

Strain engineering in Ge/GeSn core/shell nanowires

Applied Physics Letters 115, 113102 (2019); https://doi.org/10.1063/1.5111872

Performance limits of vertical GaN of conventional doped pn and natural polarization superjunction devices

Applied Physics Letters 115, 112104 (2019); https://doi.org/10.1063/1.5109389

Two-terminal terahertz detectors based on AIGaN/GaN high-electron-mobility transistors Applied Physics Letters 115, 111101 (2019); https://doi.org/10.1063/1.5114682

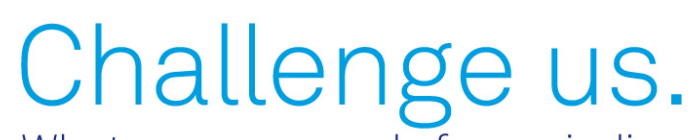

What are your needs for periodic signal detection?

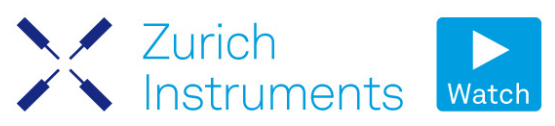

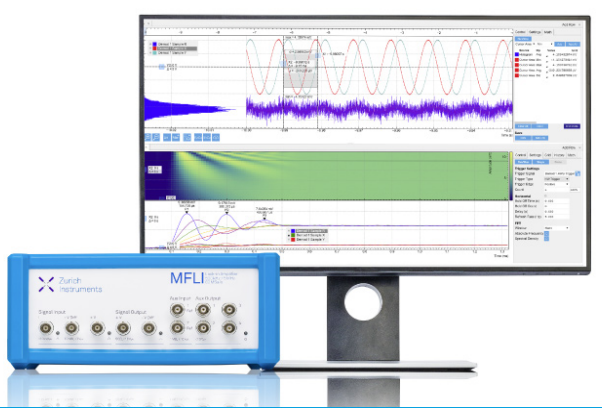

Appl. Phys. Lett. 115, 112105 (2019); https://doi.org/10.1063/1.5111018

115, 112105 


\title{
Honeycomb-serpentine silicon platform for reconfigurable electronics $\odot$
}

\author{
Cite as: Appl. Phys. Lett. 115, 112105 (2019); doi: 10.1063/1.5111018 \\ Submitted: 22 May 2019 . Accepted: 5 August 2019 . \\ Published Online: 10 September 2019
}

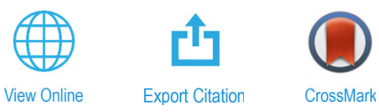

\begin{abstract}
A. N. Damdam, ${ }^{1}$ N. Qaisar, ${ }^{7}$ and M. M. Hussain ${ }^{1,2, a)}$
AFFILIATIONS

'mmh Labs, Electrical Engineering, Computer Electrical and Mathematical Science and Engineering Division, King Abdullah University of Science and Technology (KAUST), Thuwal 23955, Saudi Arabia

${ }^{2}$ Electrical Engineering and Computer Science, University of California, Berkeley, Berkeley, California 94720, USA
\end{abstract}

a)Electronic addresses: mmhussain@berkeley.edu or muhammad.hussain@kaust.edu.sa. Telephone: +966-544700072.

\begin{abstract}
The shape reconfiguration is an arising concept in advanced electronics research, which allows the electronic platform to change in shape and assume different configurations while maintaining high electrical functionality. The reconfigurable electronic platforms are attractive for state of the art biomedical technologies, where the reshaping feature increases the adaptability and compliance of the electronic platform to the human body. Here, we present an amorphous silicon honeycomb-shaped reconfigurable electronic platform that can reconfigure into three different shapes: the quatrefoil shape, the star shape, and an irregular shape. We show the reconfiguration capabilities of the design in microscale and macroscale fabricated versions. We use finite element method analysis to calculate the stress and strain profiles of the microsized honeycomb-serpentine design at a prescribed displacement of $100 \mu \mathrm{m}$. The results show that the reconfiguration capabilities can be improved by eliminating certain interconnects. We further improve the design by optimizing the serpentine interconnect parameters and refabricate the platform on a macroscale to facilitate the reconfiguration process. The macroscale version demonstrates an enhanced reconfiguration capability and elevates the stretchability by $21 \%$ along the vertical axis and by $36.6 \%$ along the diagonal axis of the platform. The resulting reconfiguring capabilities of the serpentine-honeycomb reconfigurable platform broaden the innovation opportunity for wearable electronics, implantable electronics, and soft robotics.
\end{abstract}

Published under license by AIP Publishing. https://doi.org/10.1063/1.5111018

Flexible and stretchable electronics enable significant advances in biomedical applications. The emerging internet of things (IOT) further boosts the progression of wearable and implantable devices and triggers electronic platforms with higher physical functionalities that exceed the flexibility and stretchability. ${ }^{1-3}$ To further advances in flexible and stretchable electronics, the concept of reconfiguration in the shape and size of such electronics is drawing attention. Reconfiguration is a category of physical deformation that combines stretching, bending, and twisting to transform a design into another shape. ${ }^{2,4}$ A carefully designed reconfiguration concept may realize distributed sensory networks for in vivo and in vitro biomedical devices, where the adaptability to the body curvatures and the ability to expand and change the shape without compensating the device performance may facilitate the innovation of new biomedical technologies that can be used for drug delivery, health monitoring, diagnosis, and therapeutic healing. ${ }^{5,6}$ The shape reconfiguration requires high mechanical capabilities, such as the ability to fold and elongate, which are a function of the material and design. Organic/polymeric composites are typically used to achieve stretchable and flexible electronics due to their excellent mechanical properties and cost-effectiveness. Although their electrical properties are incomparable to silicon-based electronics, many technological advancements are accomplished in display technologies by the utilization of organic/polymer-based electronics. ${ }^{7}$ Many researchers focus on the development of reconfigurable structures using different organic materials. Zhao et al. presented complex geometrical reconfigurable structures that are fabricated using thermally actuated shape memory polymers, where they show many geometrical manipulations by inducing cumulative plasticity behavior at some segments of the polymer's molecular chain and utilizing the remaining elastic segment capabilities of temporary expansion at $55^{\circ} \mathrm{C}$ and subsequent recovery to attain shape reconfiguration. ${ }^{8}$ Another approach for shape reconfiguration is inspired by the Origami and kirigami are ancient techniques for making paper-based artworks that produce 3D objects by folding/cutting 2D sheets. Many researchers have presented complex $3 \mathrm{D}$ reconfigurable structures inspired by origami and kirigami using different materials. ${ }^{9-11}$ 
Filipov et al. showed a zipper-inspired origami paper-based reconfigurable structure that has one deformation mode, where they reconfigure the design by applying a manual force. ${ }^{9}$ Lamoureux et al. present a kirigami-based structure for optical tracking by making a specific linear pattern of cuts in a flexible gallium arsenic solar cell, where they used an external-tensile actuation force, which produces buckles in the substrate while pulling it outward, for the reconfiguration purpose. ${ }^{10}$ Qaiser et al. paved the way for high-resolution stretchable displays by modeling and 3D-printing a size-variant reconfigurable platform that is mechanically guided to preserve the Light Emitting Diode (LED) pixel resolution in reconfigurable displays. ${ }^{4}$ The shape reconfiguration is also a promising feature for robotics, where an important progressing aspect in soft robotics is the shape-changing ability, which can add significant capabilities to the development of new reconfigurable platforms. ${ }^{4,6,12,13}$ Medina-Sánchez et al. presented various reconfigurable microrobots that are smart and intended to swim in biological environments. Stimuli responsive materials were used to fabricate the microrobots, and various stimuli were applied to actuate them and to precisely control their reconfigurability, where the shape reconfiguration feature was used to control the motion mechanism and speed. ${ }^{14}$ However, $90 \%$ of today's electronics utilize silicon platforms due to its suitable material properties, such as excellent semiconductor properties, fairly high electron mobility, thermal stability, and mechanical integrity. $2,5,6,13,15-17$ Nonetheless, its rigidity, fragility, and very little strain $(<1 \%)$ make the shape variations of silicon-based electronic platforms quite challenging. ${ }^{18,19}$ To address these inherent challenges, engineered stretchable geometrical designs are introduced to produce stretchable silicon platforms. For instance, Rojas et al. present a highly stretchable silicon fabric that is composed of an array of hexagons interconnected via double spiral interconnects, where the stretching capability reaches $1000 \%{ }^{5}$ Since the stretchability is an essential feature for the shape changing, employing the fractal design concept is indispensable to produce silicon-based reconfigurable electronic platforms.

In this paper, we present a honeycomb-serpentine shaped amorphous silicon ( $\mathrm{a}-\mathrm{Si}$ ) based reconfigurable electronic platform that can transform into different geometrical topologies and can be effectively utilized in the above-mentioned applications. We considered different geometries in nature and realized that an array of honeycombs could form an intriguing and effective geometry for the intended purpose of shape changing reconfiguration. The design, shown in Fig. 1(a), is characterized by three elements: the honeycomb cells, circular islands, and serpentine interconnect that constitutes all the honeycomb sides and the honeycomb island-interconnects. We integrate islands in the design to serve as a host for electronics, where the serpentine interconnects serve as mechanical support by absorbing the large deformation during stretching. They also serve as electrical interconnects between islands when they are sputtered by conductive materials. One of the unique attributes of the honeycomb geometry includes its efficiency, especially when using the unit-cell replication analogy. In this analogy, every three hexagons share one vertex and three sides, forming a net that is free of gaps. This feature provides multiple electrical interconnection options for electronics between different islands. Another important benefit of the honeycomb geometry is its large area, which is defined by

$$
\text { Area }=\frac{3 \sqrt{3}}{2} t^{2}
$$

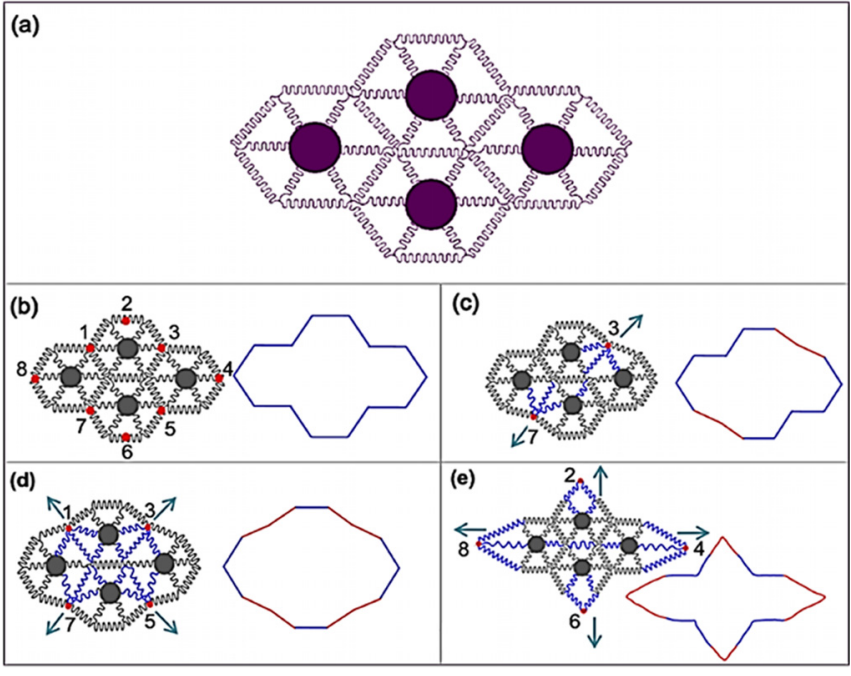

FIG. 1. (a) The serpentine-honeycomb reconfigurable platform. (b) The design with the eight reconfiguring nodes highlighted. (c) The irregular configuration. (d) The quatrefoil configuration. (e) The star configuration.

where $t$ is the side length of a regular hexagon. The large inscribed space allows for the integration of large islands and hence more electronics and greater device capabilities. The serpentine shape is one of the commonly used fractal designs in stretchable electronics, where employing that concept in the interconnects for inorganic materials minimizes the deformation seen by the platform under large strain, resulting in high stretching capabilities. The major attribute of the honeycomb-serpentine reconfigurable design is its ability to assume different irregular configurations, which can be visualized by the simultaneous stretching of certain nodes, and we call them reconfiguring nodes. Figure $1(\mathrm{~b})$ shows the eight reconfiguring nodes that we use to demonstrate the in-plane reconfiguration capability of the design. We expect to obtain three different shapes by applying a simultaneous tensile strain at different reconfiguring nodes. For instance, the simultaneous outward stretching of diagonal nodes 3 and 7 could transform the honeycomb shape into an irregular shape as shown in Fig. 1(c), while dragging-out two additional nodes, 1 and 5, could transform the irregular shape into the quatrefoil shape shown in Fig. 1(d). The platform could also be reconfigured into a star shape by stretching out the axial nodes, 2, 4, 6, and 8 simultaneously as shown in Fig. 1(e). The blue-colored serpentine interconnects in Figs. 1(c)-1(e) indicate the serpentine interconnects that are expected to attain higher stress values while stretching-out the nodes to obtain a certain configuration. To validate the reconfiguration capability, we fabricate a microscale version of the honeycomb-serpentine platform using a-Si with a platform thickness of $15 \mu \mathrm{m}$ and a serpentine interconnect width of $10 \mu \mathrm{m}$. We choose a-Si for the platform fabrication because its thin film form can be inexpensively attained using the plasma enhanced chemical vapor deposition (PECVD), which is a low temperature, highly pure, and uniform deposition process. ${ }^{20}$ Moreover, the amorphous form of silicon comes after the monocrystalline in terms of electron mobility and the semiconducting property ${ }^{20}$ and exceeds it in terms of costeffectiveness. It also has all the semiconducting properties, such as doping, photoconduction, and junction formation, and have the same 
chemical covalent bonds as the crystalline silicon permits the utilization of the same microfabrication processes for a-Si films. We started the fabrication flow by fabricating a virtual silicon on insulator (SOI) wafer with an oxide thickness of $10 \mu \mathrm{m}$ and an a-Si thickness of $15 \mu \mathrm{m}$ using PECVD as shown in Fig. S1(a). We sputtered $200 \mathrm{~nm}$ of aluminum to form a hard mask layer, coated the hard mask with positive photoresists, patterned the photoresist using the conventional lithography process, patterned the aluminum hard mask using metal reactive ion etching (RIE), and then patterned the a-Si layers using deep reactive ion etching (DRIE) as illustrated in Fig. S1(b). Next, the photoresist layer was removed using acetone, and the wafer was immersed in an aluminum wet etchant for $5 \mathrm{~min}$ to remove the aluminum hard mask from the top of the a-Si honeycomb-serpentine platforms. Scanning electron microscopy (SEM) images were produced to show the patterned structures on the virtual SOI wafer prior to the release step as shown in Fig. S2. To release the structures, the wafer was immersed in $48 \%$ hydrofluoric (HF) acid for $1 \mathrm{~h}$ at room temperature to isotopically etch the buried oxide layer. The released structures were washed using de-ionized (DI) water for four cycles, and they were placed on a regular printing paper and left to dry at room temperature as illustrated in Fig. S1(c). Figure S3 shows the released microscale honeycomb-serpentine reconfigurable platform with large diagonal and axial pads that were integrated to facilitate the reconfiguration process. The dimensions of the design are $6 \mathrm{~mm} \times 3.5 \mathrm{~mm}$.

We performed the reconfiguration test for the a-Si structures using the probe station, where we use the probes to stretch the pads outward and to fix their position. Figure 2(a) shows the fabricated honeycomb-serpentine platform in its relaxed state on the probe station. By stretching the two diagonal nodes outward, as indicated by the red arrows, we could reconfigure the design into the irregular shape as shown in Fig. 2(b). To reconfigure the design into the quatrefoil shape, we stretched-out the four diagonal nodes simultaneously, where the maximum reconfiguration achievement of the quatrefoil shape is shown in Fig. 2(c). However, the internal interconnects, colored in blue in Figs. 1(c) and 1(d), experience large deformation as

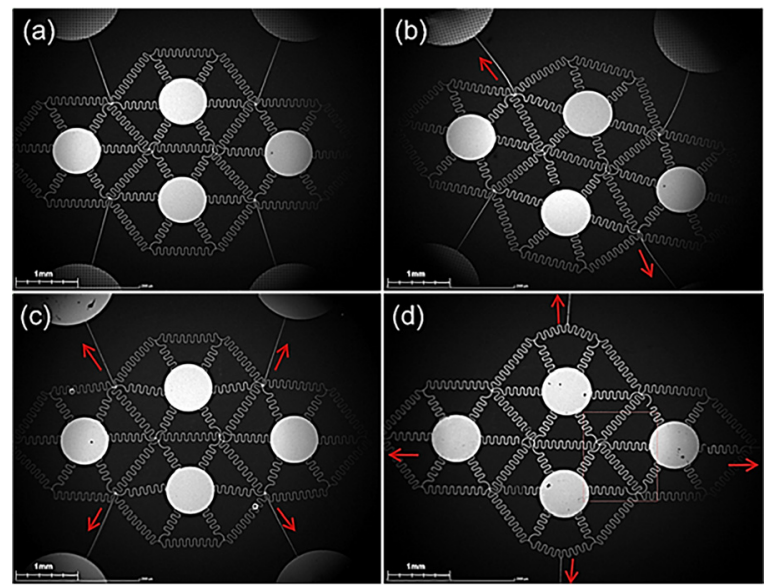

FIG. 2. (a) A microscopy image of the initially unstretched honeycomb-serpentine structure. The resultant configurations upon stretching: (b) irregular, (c) quatrefoil, and (d) star, where the nodes along with the starching direction are indicated in red arrows. expected and prevented further stretching of the shapes shown in Figs. 2 (b) and 2(c). This is attributed to the load linearity along the diagonal stretching direction, which accelerates the fracture of the internal interconnects. In contrast, the agile attempt of reconfiguring the design into the star shape by stretching the four axial nodes, as indicated by red arrows in Fig. 2(d), is ascribed to the absence of vertical internal interconnects in the vertical stretching direction, along with the starlike acute endings in the horizontal direction of the platform, which eliminates the need of a large strain along the transverse direction. Hence, experimental testing has validated our reconfiguration claim.

To investigate further the mechanical response of the design, especially the evolution of von-Mises stress as a function of prescribed displacement, we perform finite element method (FEM) analysis. The calculations are carried out up to the fracture point of the material, i.e., the shape will experience fracture exceeding these limits. Figure 3 shows the distribution of stress as a function of displacement for all the configurations. As our FEM analysis is carried out using no plasticity, the mechanical response of the material within the elastic limit requires the stress to be linearly proportional to the strain. At the same amount of displacement, i.e., $100 \mu \mathrm{m}$, the maximum stress values are 2.3 GPa for the irregular shape and $3.2 \mathrm{GPa}$ for the quatrefoil shape. The higher stress induced in the quatrefoil case occurs due to the linear distribution of stress along both diagonals of the design, where we apply the same prescribed displacement to the irregular shape but along one diagonal only, which produces less stress in the irregular configuration. Yet, a lower stress value of $2 \mathrm{GPa}$ is induced while reconfiguring the design into the star shape, which allows for the larger prescribed displacement of $155 \mu \mathrm{m}$ and higher stretchability. To validate the simulation results, we experimentally measured the largest prescribed displacement values by calculating the change in displacements upon reconfiguring the platform. This was done by measuring the lengths of the diagonal and vertical axes before and after the reconfiguration process as shown in Figs. S4(a)-S4(c). The experimental values of the maximum prescribed displacement were $100 \mu \mathrm{m}$ and $200 \mu \mathrm{m}$ along the diagonal and vertical axes, respectively. The discrepancy between the simulated and experimental maximum displacement

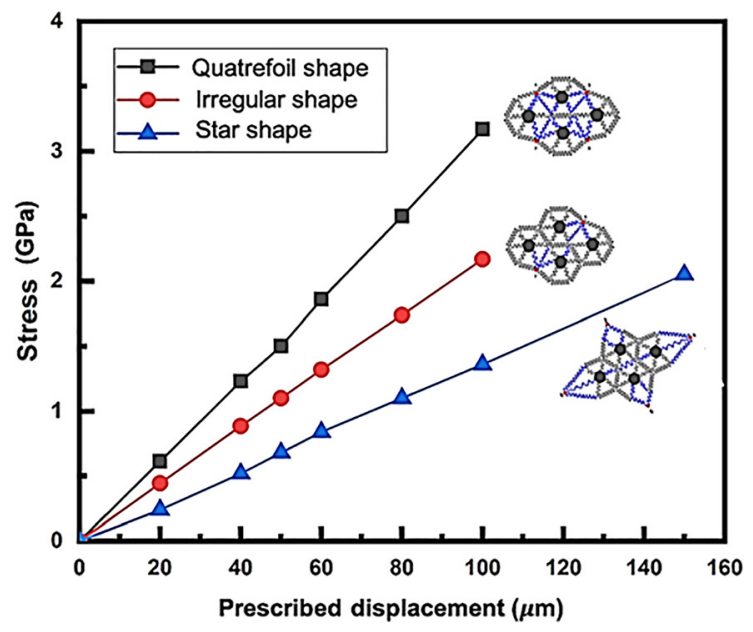

FIG. 3. The stress vs the prescribed displacement curves for the three simulated shapes. 
values for the star shape can be attributed to the $1 \%$ maximum fracture point that we assumed in our FEM analysis, which is based on the failure of silicon while following the bending theory. In other words, the actual failure point of our fabricated samples might be different since our structures experience minimal bending and mainly show the reconfigurability during stretching.

Figure 4 shows the stress and deformation profiles under a prescribed displacement of $1 \mathrm{~mm}$ at different nodes to reconfigure the honeycomb shape into the (a) irregular shape, (b) quatrefoil shape, and (c) star shape. In our FEM analysis, we take the prescribed displacement analogous to the experimentally applied stretch. Our results show that the average von Mises stress values are found to be less than $2 \mathrm{GPa}$ for all the structures, whereas we observe the maximum stress value (i.e., $2.7 \mathrm{GPa}$ ) while reconfiguring the design into the quatrefoil configuration as shown in Fig. 4(b). According to the FEM analysis, the design experiences high stress only at three serpentine interconnects: (1) the honeycomb diagonal sides that are common between two cells, (2) the internal-horizontal interconnects, and (3) the internal-diagonal interconnects that connect the islands to the honeycomb sides as highlighted in Fig. 4(b). Considering the FEM analysis stress profile, we suggest the removal of all the internal-horizontal interconnects to improve the stretchability of the structure while keeping the internal-diagonal ones to associate the islands with the honeycombs. Although the diagonal interconnects are experiencing high von Mises stress as well, we did not omit them to retain the honeycomb unit cell topology, which is crucial for the cells' replication

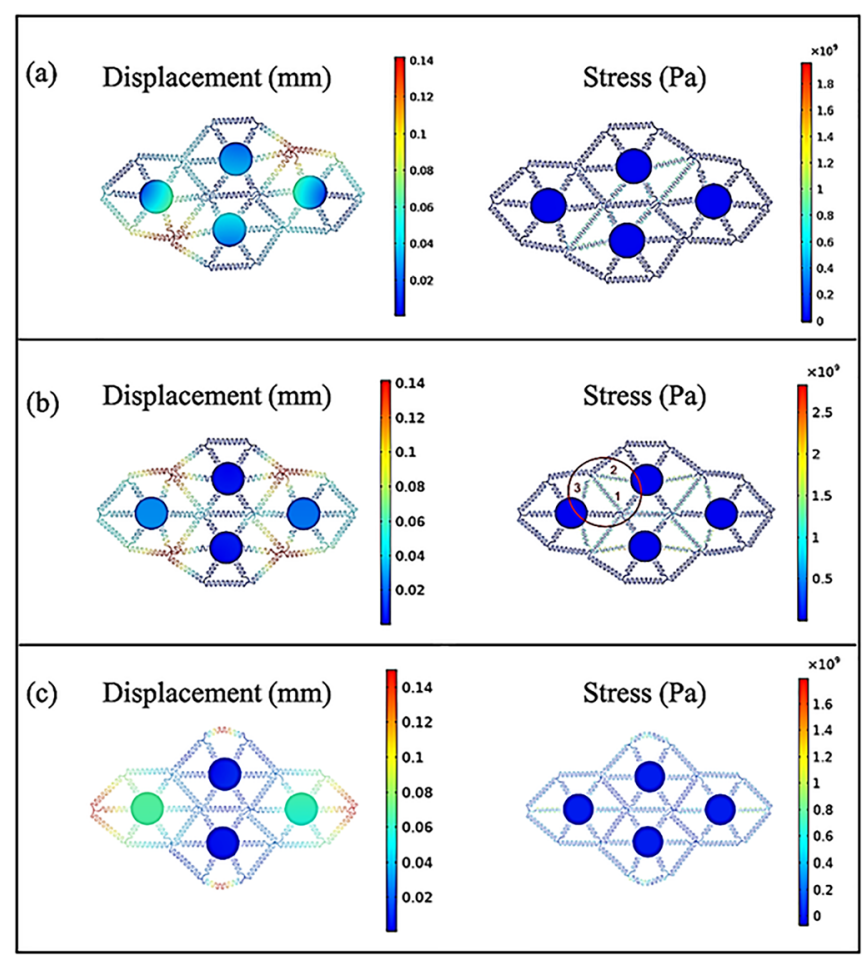

FIG. 4. The prescribed displacement and stress profiles for (a) the irregular shape, (b) the quatrefoil shape, and (c) the star shape. feature. Additionally, the diagonal interconnects would offer essential electrical connections between islands in different cells.

Next, we optimize the serpentine dimensions to further improve the stretchability. Three main elements affect the mechanical deformation and stretchability of the serpentine interconnects, which are the aspect ratio $w / t$, the number of unit cells $(\mathrm{m})$, and the ratio of the spacing between two arms $l_{1}$ and the arm length $l_{2}$, as illustrated in Fig. S5. Increasing $l_{2} / l_{1}$ and the number of unit cells $(\mathrm{m})$ while reducing $w / t \leq(1 / 2)$ improves the stretchability and reduces the out-ofplane deformation of structures that are fabricated using high Young's modulus materials, such as silicon. ${ }^{19,21}$ Taking that into consideration, we optimize the serpentine unit cell parameters as follows: we decrease the aspect ratio $w / t$ from $15 \mu \mathrm{m} / 10 \mu \mathrm{m}$ to $10 \mu \mathrm{m} / 20 \mu \mathrm{m}$ and increase $l_{2} / l_{1}$ from $80 \mu \mathrm{m} / 50 \mu \mathrm{m}$ to $80 \mu \mathrm{m} / 10 \mu \mathrm{m}$. In addition, we scaled-up the honeycomb design from a microscale to a macroscale to facilitate the fabrication and the manual reconfiguration processes and to elevate the stretchability, where increasing the side length of the honeycomb shape allows us to have more serpentine unit cells (m), which improve the stretchability further. Table I shows a comparison between the initial and the optimized parameters of the serpentine interconnects.

We refabricated the design on a macroscale considering the optimized parameters, where the dimensions of the optimized platform are $2.5 \mathrm{~cm} \times 1.8 \mathrm{~cm}$. Figure 5 (a) shows the fabricated macroscale honeycomb-serpentine platform before stretching. The reconfiguration capability of the optimized design was enhanced as shown in Figs. 5(b)-5(d), where the applied displacement to obtain the irregular and quatrefoil shapes is $15 \mathrm{~mm}$ and the stretchability is experimentally measured along the diagonal axes to be $4.7 \%$. In contrast, the stretchability along the vertical direction is measured to be $14.2 \%$ while applying a displacement of $25 \mathrm{~mm}$ to reconfigure the design into the star shape as shown in Fig. 5(d). The stretchability measurement was governed by the following formula:

$$
\text { Stretchibility }=\frac{L_{2}-L_{1}}{L_{1}} \times 100,
$$

where $L_{1}$ is the axis length before stretching and $L_{2}$ is the axis length after stretching. The lower diagonal stretchability can be attributed to the induced high stress in the diagonal interconnects. To further quantify the optimization effect, we experimentally calculate the stretchability of the microscale structure shown in Fig. 2, where the stretchability measurements for both the structures are displayed in Table I. In comparison, the parameter optimization of the serpentine interconnects elevates the stretchability along the diagonal and vertical axes by $36.6 \%$ and $21.3 \%$, respectively, which enhances the reconfiguration capabilities of design. Additionally, the omission of the internalhorizontal interconnects increases the mechanical performance of the irregular and quatrefoil shapes, leading to a significant enhancement in the shape reconfiguration capacity that enables us to exactly mimic the configurations envisioned in Fig. 1.

In summary, we have introduced a concept of reconfigurability by using the honeycomb design. The design proved its capability of reforming into different shapes by stretching and smoothly recovering into the original honeycomb shape. The design maintained its mechanical stability during the reconfiguration process; although it was fabricated using the highly fragile a-Si, it was able to sustain high prescribed displacement values that reached $155 \mu \mathrm{m}$. The maximum 
TABLE I. A comparison between the initial and the optimized serpentine unit cell parameters and the resultant stretchability along the diagonal and vertical axes of the platform.

\begin{tabular}{lcr}
\hline \hline Parameters & Initial parameters & Optimized parameters \\
\hline Arm length $\left(l_{2}\right) /$ spacing $\left(l_{1}\right)$ & $80 \mu \mathrm{m} / 50 \mu \mathrm{m}=1.6$ & $80 \mu \mathrm{m} / 10 \mu \mathrm{m}=8$ \\
Number of cells $(\mathrm{m})$ & $5-9$ for different springs lengths & $\geq 29$ \\
Aspect ratio $(w / t)$ & $15 \mu \mathrm{m} / 10 \mu \mathrm{m}=0.66$ & $10 \mu \mathrm{m} / 20 \mu \mathrm{m}=0.5$ \\
Tested diagonal stretchability & $3.44 \%$ & $4.7 \%$ \\
Tested vertical stretchability & $11.7 \%$ & $14.2 \%$ \\
\hline \hline
\end{tabular}

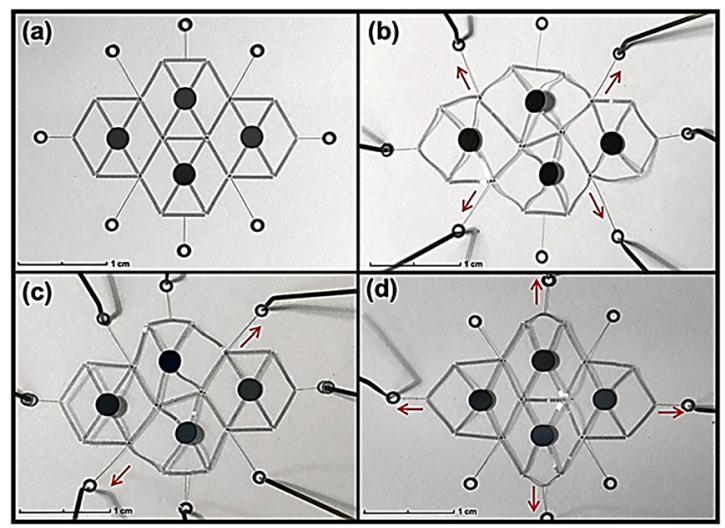

FIG. 5. (a) The fabricated macroscale version of the a-Si honeycomb-serpentine reconfigurable platform. The resultant configurations upon stretching the structure as indicated by red arrows: (b) the quatrefoil shape, (c) the irregular shape, and (d) the star shape.

stretchability of the design was found to be around $14.2 \%$; however, its reconfiguration capability can be further improved by employing ultrastretchable fractal interconnects. Furthermore, the obtained stretchability of the honeycomb-serpentine reconfigurable platform is adequate for the applications that demand little strain capabilities. For instance, the human skin can be stretched up to $20 \%$, and so our design can be potentially integrated into wearable stretchable electronics devices.

See the supplementary material for the microfabrication flow illustration, SEM imaging of the microscale amorphous-silicon honeycomb design prior to the release step, a picture for the released structures showing the axial and diagonal pads, and the experimental measurement of the vertical and diagonal displacements in addition to the serpentine unit cell parameters.

\section{REFERENCES}

1J. M. Nassar, J. P. Rojas, A. M. Hussain, and M. M. Hussain, Extreme Mech. Lett. 9, 245-268 (2016).

${ }^{2}$ A. C. Cavazos Sepulveda, M. S. Diaz Cordero, A. A. A. Carreno, J. M. Nassar, and M. M. Hussain, Appl. Phys. Lett. 110(13), 134103 (2017).

${ }^{3}$ A. M. Hussain and M. M. Hussain, Adv. Mater. 28(22), 4219-4249 (2016).

${ }^{4}$ N. Qaiser, S. M. Khan, K. Chow, M. D. Cordero, I. Wicaksono, and M. M. Hussain, Adv. Mater. Technol. 3(12), 134103 (2018).

5. P. Rojas, A. Arevalo, I. G. Foulds, and M. M. Hussain, Appl. Phys. Lett. 105(15), 154101 (2014).

${ }^{6}$ N. Qaiser, S. M. Khan, M. Nour, M. U. Rehman, J. P. Rojas, and M. M. Hussain, Appl. Phys. Lett. 111(21), 214102 (2017).

${ }^{7}$ M. U. Chaudhry, K. Muhieddine, R. Wawrzinek, J. Li, S. C. Lo, and E. B. Namdas, ACS Photonics 5(6), 2137-2144 (2018).

${ }^{8}$ Q. Zhao, W. K. Zou, Y. W. Luo, and T. Xie, Sci. Adv. 2(1), e1501297 (2016).

${ }^{9}$ E. T. Filipov, T. Tachi, and G. H. Paulino, Proc. Natl. Acad. Sci. U. S. A. 112(40), 12321-12326 (2015)

${ }^{10}$ A. Lamoureux, K. Lee, M. Shlian, S. R. Forrest, and M. Shtein, Nat. Commun. 6, 8092 (2015).

${ }^{11}$ X. Ning, X. J. Wang, Y. Zhang, X. E. Yu, D. Choi, N. Zheng, D. S. Kim, Y. G. Huang, Y. H. Zhang, and J. A. Rogers, Adv. Mater. Interfaces 5(13), 1800284 (2018).

${ }^{12}$ B. Mazzolai, L. Margheri, M. Cianchetti, P. Dario, and C. Laschi, Bioinspiration Biomimetics 7(2), 025005 (2012).

${ }^{13}$ N. Qaiser, S. M. Khan, and M. M. Hussain, J. Appl. Phys. 124(3), 034905 (2018).

${ }^{14}$ M. Medina-Sanchez, V. Magdanz, M. Guix, V. M. Fomin, and O. G. Schmidt, Adv. Funct. Mater. 28(25), 1707228 (2018).

${ }^{15}$ G. A. T. Sevilla, S. Bin Inayat, J. P. Rojas, A. M. Hussain, and M. M. Hussain, Small 9(23), 3916-3921 (2013).

${ }^{16}$ D. H. Kim, R. Ghaffari, N. S. Lu, and J. A. Rogers, Annu. Rev. Biomed. Eng. 14, 113-128 (2012)

${ }^{17}$ J. P. Rojas, G. A. T. Sevilla, M. T. Ghoneim, S. Bin Inayat, S. M. Ahmed, A. M. Hussain, and M. M. Hussain, ACS Nano 8(2), 1468-1474 (2014).

${ }^{18}$ J. A. Fan, W. H. Yeo, Y. W. Su, Y. Hattori, W. Lee, S. Y. Jung, Y. H. Zhang, Z. J. Liu, H. Y. Cheng, L. Falgout, M. Bajema, T. Coleman, D. Gregoire, R. J. Larsen, Y. G. Huang, and J. A. Rogers, Nat. Commun. 5, 3266 (2014).

${ }^{19}$ T. Widlund, S. X. Yang, Y. Y. Hsu, and N. S. Lu, Int. J. Solids Struct. 51(23-24), 4026-4037 (2014).

${ }^{20}$ Y. Hamakawa, Appl. Surf. Sci. 22(3), 859-878 (1985).

${ }^{21}$ Y. H. Zhang, S. Xu, H. R. Fu, J. Lee, J. Su, K. C. Hwang, J. A. Rogers, and Y. G. Huang, Soft Matter 9(33), 8062-8070 (2013). 